\title{
Determinantes de escolha alimentar ${ }^{1}$
}

\section{Food choice factors}

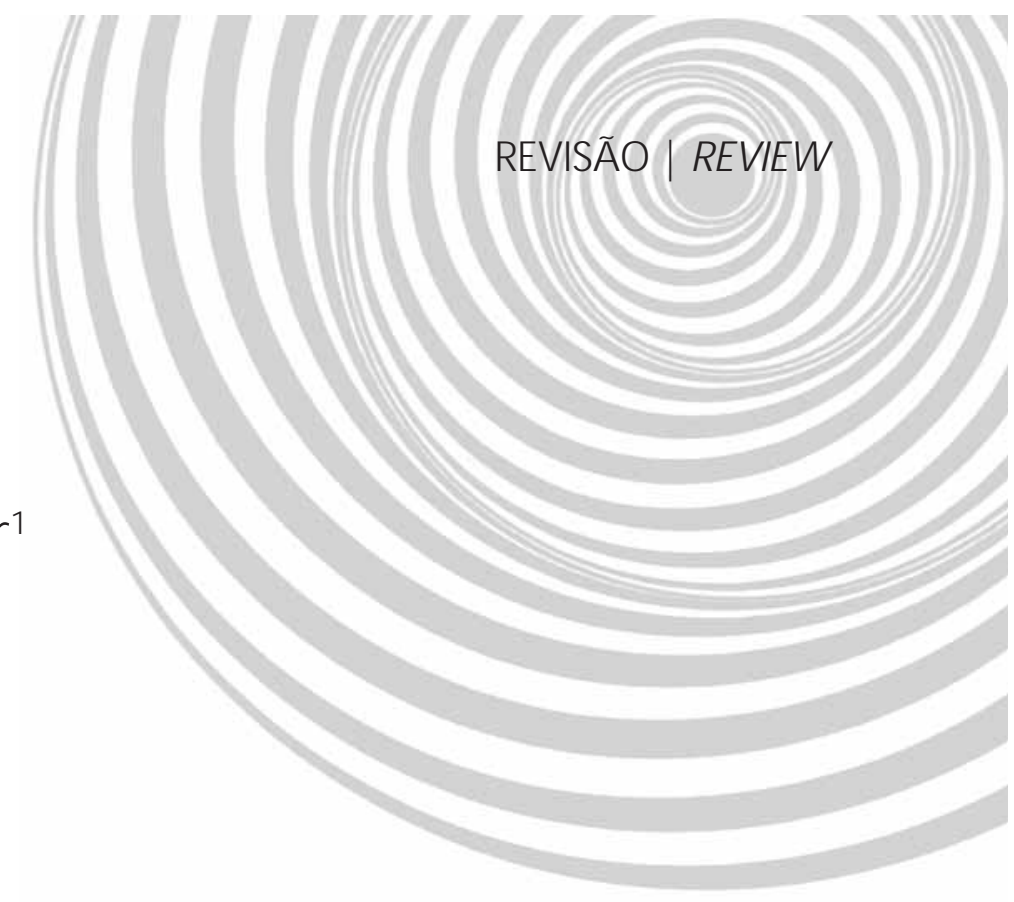

\author{
Manuela Mika JOMORI \\ Rossana Pacheco da Costa PROENÇA² \\ Maria Cristina Marino CALVO ${ }^{3}$
}

\section{RE S U M O}

A escolha alimentar humana está baseada, por um lado, na condição onívora do homem, isto é, apresentar a capacidade de comer de tudo e, por outro lado, por diversos outros fatores que irão influenciar o indivíduo nessa decisão. Buscando discutir esses determinantes, realizou-se uma revisão da literatura científica sobre a questão. Foram identificados alguns estudos e reflexões sobre determinantes da escolha alimentar humana em diferentes contextos, além de livros que discutiam sobre o tema. Para viabilizar a discussão, incluiu-se uma reflexão inicial sobre a condição onívora do homem, seguindo-se os determinantes relacionados com os alimentos e aqueles relacionados com o comedor. Assim, identificaram-se variáveis relacionadas aos alimentos como o preço, o sabor, a variedade, o valor nutricional, a aparência e a higiene, por exemplo. Já as variáveis relacionadas ao próprio indivíduo foram divididas em determinantes biológicos, sócio-culturais e antropológicos, bem como econômicos e psicológicos. Para finalizar, discute-se sobre a interação desses determinantes em métodos qualitativos e quantitativos para avaliar a escolha alimentar humana. Destaca-se que o cruzamento de olhares interdisciplinar entre as Ciências Humanas e as Ciências Nutricionais pode auxiliar no aprofundar de conhecimentos sobre o comedor humano.

Termos de indexação: Alimentos. Alimentação coletiva. Comportamento alimentar. Escolha. Pesquisa interdisciplinar.

\section{A B S T R A C T}

Human food choice is based, on the one hand, on the omnivorous condition of man, that is, to be able to eat everything, and on the other hand, by many other factors that will influence the individual in this decision. In an attempt to discuss these determinants, a scientific literature review was done on the issue. Some studies and reflections on determinants of human food choice in different contexts, besides books that discuss the

\footnotetext{
${ }^{1}$ Artigo elaborado a partir da dissertação de M.M. JOMORI, intitulada "Escol ha al imentar do comensal de um restaurante por peso". Universidade Federal de Santa Catarina; 2006. Apoio: Universidade Federal de Santa Catarina, - Programa de Apoio à Pós-Graduação, Coordenação de Aperfeiçoamento de Pessoal de Nível Superior.

2 Universidade Federal de Santa Catarina, Programa de Pós-Graduação em Nutrição, Núcleo de Pesquisa de Nutrição em Produção de Refeições. Campus Universitário, Trindade, 88040-900, Florianópolis, SC, Brasil. Correspondência para/Correspondence to: R.P.C. PROENÇA. E-mail: «mikajomori@yahoo.com.br>.

${ }^{3}$ Departamento de Saúde Pública, Universidade Federal de Santa Catarina. Florianópolis, SC, Brasil.
} 
theme were identified. In order to make the discussion viable, an initial reflection on the omnivorous condition of man was included, followed by determinants associated with foods and those associated with the eater. Thus, variables associated with foods such as price, flavor, variety, nutritional value, appearance and hygiene were identified. The variables associated with the individual were divided into biological, sociocultural and anthropologic determinants, as well as economic and psychological determinants. Finally, the interaction of these determinants is discusses in qualitative and quantitative methods to assess the human food choice. We point out that the sharing of interdisciplinary knowledge between the Human and Nutritional sciences can help to deepen the knowledge on the human eater.

Indexing terms: Foods. Collective feeding. Feeding behavior. Choice. Interdisciplinary research.

\section{N T R O D U Ç Ã O}

$\mathrm{O}$ ato alimentar, segundo Poulain ${ }^{1}$, se desenrola de acordo com regras impostas pela sociedade, influenciando a escolha alimentar. Essas regras são representadas pelas maneiras no preparo dos alimentos, pela montagem dos pratos e pelos rituais das refeições (como, por exemplo, os modos e as posições das pessoas à mesa, a divisão da comida entre os indivíduos, os horários estipulados, entre outros), contribuindo para que o homem se identifique com o alimento, também por sua representação simbólica. Nessa relação, pode-se destacar a questão do homem como um ser vivo onívoro, representada pela capacidade de comer de tudo, que lhe dá uma suposta liberdade de escolha alimentar. Entretanto, nem tudo é escolhido por ele, uma vez que o indivíduo é determinado por diversos fatores, que irão pesar nessa decisão. Esses fatores podem englobar 0 meio ambiente, o qual está relacionado aos recursos disponíveis e aos relacionamentos sociais, bem como a história individual. Essas condições permitirão ao homem refletir sobre o que vai comer.

Assim, considerando os valores do grupo social no qual o indivíduo está inserido, a construção de identidades culturais e a diferenciação individual, o comedor humano ${ }^{(4)}$ seleciona os recursos naturais disponíveis e os transforma em preparações culinárias para poder consumi-los e atender às suas necessidades. Esses parâmetros conduzem à tomada de decisão pelo homem com relação à sua alimentação².
Uma vez que a escolha alimentar é um processo complexo que envolve fatores sócio-culturais e psicológicos ${ }^{3}$ e, dada a indiscutível importância deste tema para os estudiosos em alimentação de variadas áreas do conhecimento, Poulain \& Proença ${ }^{4,5}$ discutem sobre a necessidade de reflexão de abordagens metodológicas pluridisciplinares na tentativa de melhorar a compreensão desse processo.

Nesse sentido, este texto apresenta uma revisão sobre alguns estudos que investigaram a escolha alimentar humana, visando refletir sobre os seus determinantes. Para viabilizar a discussão, apresentam-se os determinantes relacionados com o comedor, iniciando com uma reflexão sobre a condição onívora do homem, seguindo-se os determinantes relacionados com os alimentos. Finalizando, discute-se sobre a interação desses determinantes em alguns métodos, para avaliar a escolha alimentar humana, que podem ser utilizados em diversos contextos.

A pesquisa foi feita utilizando-se as bases de dados Medline, SciELO e Portal da Capes, considerando publicações a partir do ano de 1999, utilizando-se as palavras-chave: escolha alimentar, comportamento alimentare alimentação, nas línguas portuguesa e inglesa. Foram identificados alguns estudos que citavam determinantes da escolha alimentar humana em diferentes contextos, além de livros que discutiam a questão.

\footnotetext{
${ }^{4}$ A palavra comedor, segundo nota de tradução do livro Sociologias da Alimentação, de Poulain (p.22), em uma tradução literal da língua francesa da palavra mangeur, significa o ser que come, adotado pela sociologia da alimentação atual no sentido de distinguir do termo comensal, que significa o ser que come com outras pessoas à mesma mesa.
} 


\section{Variáveis relacionadas ao comedor}

O homem onívoro, na sua liberdade de escolha alimentar, procura separar o elemento comestível do não-comestível ${ }^{6,7}$. A alimentação humana é referida como um fato em que se podem verificar as necessidades de ordem biológica, bem como os desejos do comedor, que podem ser social e culturalmente definidos. Assim, mesmo na condição onívora, em que o homem é capaz de consumir biologicamente de tudo, as escolhas alimentares se baseiam nos sistemas culturais dos grupos humanos, os quais só se permitem alimentar-se do que é aceito culturalmente. Isso torna a alimentação humana ambivalente, pois, segundo Fischler ${ }^{6}$, ela fica marcada pela contradição entre a neofilia e a neofobia alimentares. A neofilia alimentar é a "tendência à exploração, necessidade de mudança, de novidade e de variedade" , e a neofobia alimentar está relacionada à prudência, ao receio do desconhecido e à resistência à inovação, afetando a sua escolha alimentar.

Ao mesmo tempo, o indivíduo se depara com seus anseios alimentares, envolvendo o gosto e suas especificidades na seleção dos alimentos. De acordo com Fischler ${ }^{6}$, o gosto, inserido na dimensão hedônica, compreende a interio rização da informação cultural, a qual busca ajustá-la às regras culinárias. Essa transmissão das estruturas culturais da alimentação se dá desde a infância, não sendo, necessariamente, realizada pelo ensinamento direto dos pais para os filhos. A formação do gosto na infância é devida ao processo de aprendizagem, ou seja, a criança observa o que outro indivíduo faz e tenta imitá-lo. Esse processo, ao se repetir no cotidiano dos grupos sociais, permite contribuir para a formação das preferências alimentares desde a infância. Dessa forma, qualquer indivíduo está susceptível à influência social para a adaptar a seus gostos e, conseqüentemente, às suas escolhas alimentares.

Segundo Bourdieu ${ }^{8}$, há o gosto de necessidade, caracterizado pelo atendimento da sacieda- de e pela adequação ao menor custo, e o gosto de luxo, que se distancia da necessidade pela seleção alimentar mais refinada e pela ascensão social, buscando afastar-se da camada social de origem. Essa distinção contribui para caracterizar as diferenças entre as classes sociais, com o primeiro tipo de gosto incidindo mais sobre as camadas populares. Mas essas características podem ser conferidas pelo fato de as pessoas optarem por alimentos que Ihes dêem status social.

Nesse sentido, a escolha alimentar de um indivíduo está relacionada aos fatores do meio ambiente, da história individual e da personalidade refletida em valores pessoais ${ }^{7}$. Considerando que a escolha alimentar é um processo dinâmico e é construída por diversos determinantes, discutiremos as variáveis de escolha relacionadas aos alimentos e ao comedor humano.

As escolhas alimentares são construídas por um sistema normativo imposto ao homem, denominado por Fischler ${ }^{6}$ como utopia alimentar. Nesse conceito, o autor faz uma associação dos termos ração ${ }^{(5)}$ e razão, que está relacionada ao custo-benefício da alimentação dentro de regras que irão controlar o modo alimentar dos indivíduos. Isso se refere ao peso dos desejos em contraposição às regras que dão ao alimento certo poder de manipulação social do indivíduo, o qual é regido por normas no momento da escolha do alimento.

Com relação aos fatores individuais, nos estudos encontrados eles foram classificados como variáveis relacionadas aos indivíduos, conforme 0 demonstrado no Quadro 1.

Gedrich ${ }^{9}$ divide o comportamento alimentar em cinco grandes determinantes: biológicos, sócio-culturais, antropológicos, econômicos, psicológicos. 0 determinante biológico é subdividido em componentes fisiológicos, patológicos e genéticos, sendo os primeiros componentes conferidos pelas necessidades energéticas e de nutrientes para manutenção do metabolismo orgânico. 0 componente genético, de acordo com Fantino ${ }^{10}$,

\footnotetext{
${ }^{5}$ Ração se refere à comida.
} 
Quadro 1. Variáveis relacionadas ao comensal, de acordo com os autores pesquisados.

\begin{tabular}{|c|c|c|c|c|c|c|c|c|c|}
\hline $\begin{array}{l}\text { Variáveis do } \\
\text { indivíduo }\end{array}$ & Fischler ${ }^{6}$ & Furst et al. ${ }^{15}$ & Fantino $^{14}$ & Niel et al. ${ }^{10}$ & ${ }^{0}$ Poulain ${ }^{12}$ & $\begin{array}{l}\text { Courbeau } \\
\& \text { Poulain }\end{array}$ & Tse et al. ${ }^{13}$ & ${ }^{3}$ Gedrich $^{9}$ & Batalla et al. ${ }^{19}$ \\
\hline \multicolumn{10}{|l|}{ Biológicas } \\
\hline Idade & & $x$ & $x$ & & $x$ & & & & $x$ \\
\hline Sexo & & $x$ & $x$ & & $x$ & & & $x$ & $x$ \\
\hline Estado de saúde & & $x$ & $x$ & & $x$ & & & $x$ & \\
\hline \multicolumn{10}{|c|}{ Sócio-culturais e antropológicas } \\
\hline Étnico-cultural & $x$ & $x$ & & & $x$ & $x$ & & $x$ & $x$ \\
\hline Ambiente social & $x$ & $x$ & & $x$ & $x$ & $x$ & $x$ & $x$ & $x$ \\
\hline Riscos alimentares & $x$ & $x$ & & & $x$ & $x$ & & & \\
\hline Estética corporal & $x$ & & & & $x$ & $x$ & & & \\
\hline Curiosidade & $x$ & & & & $x$ & $x$ & & & \\
\hline Hedonismo & $x$ & $x$ & $x$ & & & $x$ & & & \\
\hline \multicolumn{10}{|l|}{ Econômicas } \\
\hline Preço & & $x$ & & $x$ & & & $x$ & $x$ & $x$ \\
\hline
\end{tabular}

é um determinante que pode atuar sobre a sensibilidade gustativa, indiretamente sobre a palatabilidade e, conseqüentemente, sobre as preferências alimentares. Pode-se considerar a influência do sexo, da fase da vida, da sensibilidade sensorial às substâncias específicas e da palatabilidade como determinantes biológicos. Cabe ressaltar aqui a questão do determinante cultural do gosto, abordada por Bourdieu ${ }^{8}$, ao se referir ao gosto de necessidade, o qual está intimamente ligado à saciedade, bem como ao gosto de luxo, referindo-se ao gosto refinado, das camadas mais altas, que as distingue das demais.

Nesse sentido, é viável abordar os determinantes sócio-culturais na alimentação também referidos por Falk et al. ${ }^{3}$, Furst et al. ${ }^{11}$ e por Falk et al. ${ }^{12}$. Esses determinantes incluem a questão do convivio do indivíduo (família, amigos, relações de trabalho...), dos grupos a que gostaria de pertencer e de ser aceito (status social, identificação com o grupo) ou do grupo ao qual o indivíduo não deseja estar associado (distinção). Nesse contexto, verificam-se as influências das normas de um grupo sobre o comportamento alimentar dos indivíduos ${ }^{2,9,12}$. É possível sugerir que o determinante sócio-cultural tem caminhado junto aos outros, uma vez que ele é abordado em todos os outros fatores, destacando os determinantes antropológicos e psicológicos. 0 determinante antropológico refere-se à liberdade de escolha alimentar do indivíduo em paralelo às representações do que ele prefere consumir6,7,9. Outras abordagens, nesse contexto, também são citadas para interpretar a escolha alimentar individual englobando variáveis como: valores, confiança, crenças ou expectativas, intenções, envolvimentos e experiências ${ }^{9}$.

Os determinantes econômicos são referidos ao poder de compra do indivíduo e à condição da oferta e estão associados aos fatores demográficos, no contexto dos lares como unidades de consumo, em que é possível estratificar os grupos por região, de acordo com a situação econômica ${ }^{9}$.

Para avaliar atributos que tornam um restaurante lotado, Tse et al. ${ }^{13}$ realizaram 0 estudo com 30 indivíduos em Hong Kong com relação aos restaurantes chineses. Três fatores foram citados: alta qualidade, baixo preço e boa reputação do estabelecimento. A partir disso, foram definidas três hipóteses: “1) os clientes atribuem alta qualidade a um restaurante lotado e baixa qualidade a um restaurante vazio; 2 ) a um restaurante lotado os clientes atribuem boa reputação, ao vazio atribuem a má reputação; 3) a um restaurante lotado os clientes atribuem ao baixo preço, enquanto o vazio é devido ao preço alto". Para medir as declarações dos indivíduos e as suas percepções nas diversas situações citadas, 
foi realizado um questionário em escalas de sete pontos com opções desde forte discordância até forte concordância. Para testar cada hipótese, testes foram feitos para determinar se a primeira percepção afeta as demais, e se verificou a confiabilidade das declarações de todas as percepções. Obtiveram-se escalas confiáveis de medidas das percepções da qualidade da comida, da reputação do restaurante e do preço. Todas as percepções foram, portanto, utilizadas como variáveis dependentes e a percepção da lotação do estabelecimento como variável independente em todas as situações. Isso conferiu que a lotação de um restaurante foi atribuída à qualidade da comida, à reputação do estabelecimento e ao preço, segundo os sujeitos pesquisados.

Cabe ressaltar uma pesquisa realizada no Brasil por Batalha et al. ${ }^{14}$, sobre a percepção pelos indivíduos do preço de certos produtos alimentícios com relação à sua renda. Constatou-se que a percepção do preço dos alimentos em geral é considerada cara pela maioria dos consumidores entrevistados, nos diferentes níveis de renda. Diante dessa análise, feita pelos autores, as variáveis de preço e renda influenciam nas decisões de compra, entretanto, não podem ser consideradas como variáveis isoladas para uma interpretação do comportamento do consumidor nem como a proporcionalidade entre renda e consumo.

Os motivos da escolha e das práticas alimentares são justificados por diversas variáveis, inseridas em uma ordem lógica, que escapam à consciência do indivíduo ${ }^{15}$. A escolha alimentar é um processo multideterminado ${ }^{3,11,12}$, que inclui um sistema pessoal dinâmico e construído ${ }^{15,16}$.

Furst et al. ${ }^{11}$ propuseram uma abordagem construtivista para desenvolver um modelo de múltipla perspectiva do processo de escolha alimentar, também utilizada por Falk et al. ${ }^{12}$. Essa equipe de pesquisadores entrevistou sujeitos em lojas e outros pontos de vendas de alimentos, durante as compras, utilizando técnicas qualitativas, gravadas e transcritas para análises posteriores. A abordagem foi realizada no momento da escolha dos alimentos pelos sujeitos, encorajando-os a salientarem estratégias de compras, preferências alimentares, mudanças nos hábitos alimentares, bem como temas que surgiram durante a entrevista. Assim, pôde-se identificar e discutir os conceitos e as categorias emergentes das transcrições das entrevistas realizadas, além do uso do método comparativo para implementar uma abordagem teorética para a análise dos dados. Definiram-se então as categorias, examinando suas inter-relações. Essa abordagem permitiu aos pesquisadores enfocar alguns critérios de escolha alimentar em diversas situações e locais.

Com base nisso, Furst et al. ${ }^{11}$ desenvolveram o modelo teórico que ilustra o processo da escolha alimentar, visualizado no esquema da Figura 1. Nesse modelo, existem três grandes componentes: o curso de vida, as influências e o sistema pessoal. 0 primeiro inclui o papel pessoal e o meio ambiente sócio-cultural ao qual o indivíduo foi exposto. As influências são baseadas no primeiro componente e envolvem cinco fatores, que emergiram durante o estudo: os ideais, os individuais, os recursos disponíveis, a estrutura social e o contexto alimentar. Os ideais consistem nas expectativas, nas crenças e nos padrões, como pontos de referências e comparação para avaliar a escolha alimentar de cada indivíduo. Os fatores individuais são baseados nas necessidades e nas preferências pessoais, tanto de ordem psicológica (gostos/aversões, estilos alimentares pessoais e emoções) quanto fisiológica (sexo, idade, estado de saúde, preferências sensoriais - sensibilidade gustativa e estado de fome - saciedade). Os recursos disponíveis referem-se aos recursos palpáveis, como o dinheiro, os equipamentos e o espaço físico, e aos recursos não-palpáveis como as habilidades e os conhecimentos técnicos e o tempo. A estrutura social possui dimensões importantes como a natureza das relações interpesssoais (família, convívio doméstico, local de trabalho e eventos especiais), os papéis e os significados sociais. Finalmente, o contexto alimentar limita-se ao ambiente físico, condição social do local, fatores do fornecimento de alimentos (tipos, fontes 
e disponibilidade de alimentos no sistema alimentar, incluindo fatores sazonais e de mercado). Esta última influência está diretamente relacionada à estrutura social, a qual provê um ambiente para a escolha alimentar, que define comportamentos específicos, em que o alimento é fornecido por um sistema social alimentar.

A partir disso, chega-se a um elo para formar o terceiro componente do modelo - o sistema pessoal - que engloba o processo de negociação de valores e uma série de estratégias definidas para fazer a escolha alimentar. 0 processo de negociação de valores consiste no balanço de valores que o indivíduo faz em uma determinada situação de escolha alimentar. Nessa etapa do processo de escolha alimentar, o indivíduo pode levar em consideração: os aspectos sensoriais; os fatores econômicos; a conveniência, destacando a relação custo e benefício, considerando o gasto com o tempo e a comodidade; a saúde e a nutrição, relacionadas com a prevenção ou o controle de doenças, controle de peso e bem-estar corporal; a organização dos relacionamentos, considerando as preferências e as necessidades das pessoas com quem o indivíduo convive; $\mathrm{e}$ a qualidade (refere-se à busca por um padrão de excelência). Dessa forma, o indivíduo desenvolve as estratégias para chegar a uma escolha alimentar.

Outro estudo sobre escolha alimentar, que se baseou nesse modelo, concluiu que o sistema alimentar pessoal leva em consideração a impor-

CURSO DE VIDA
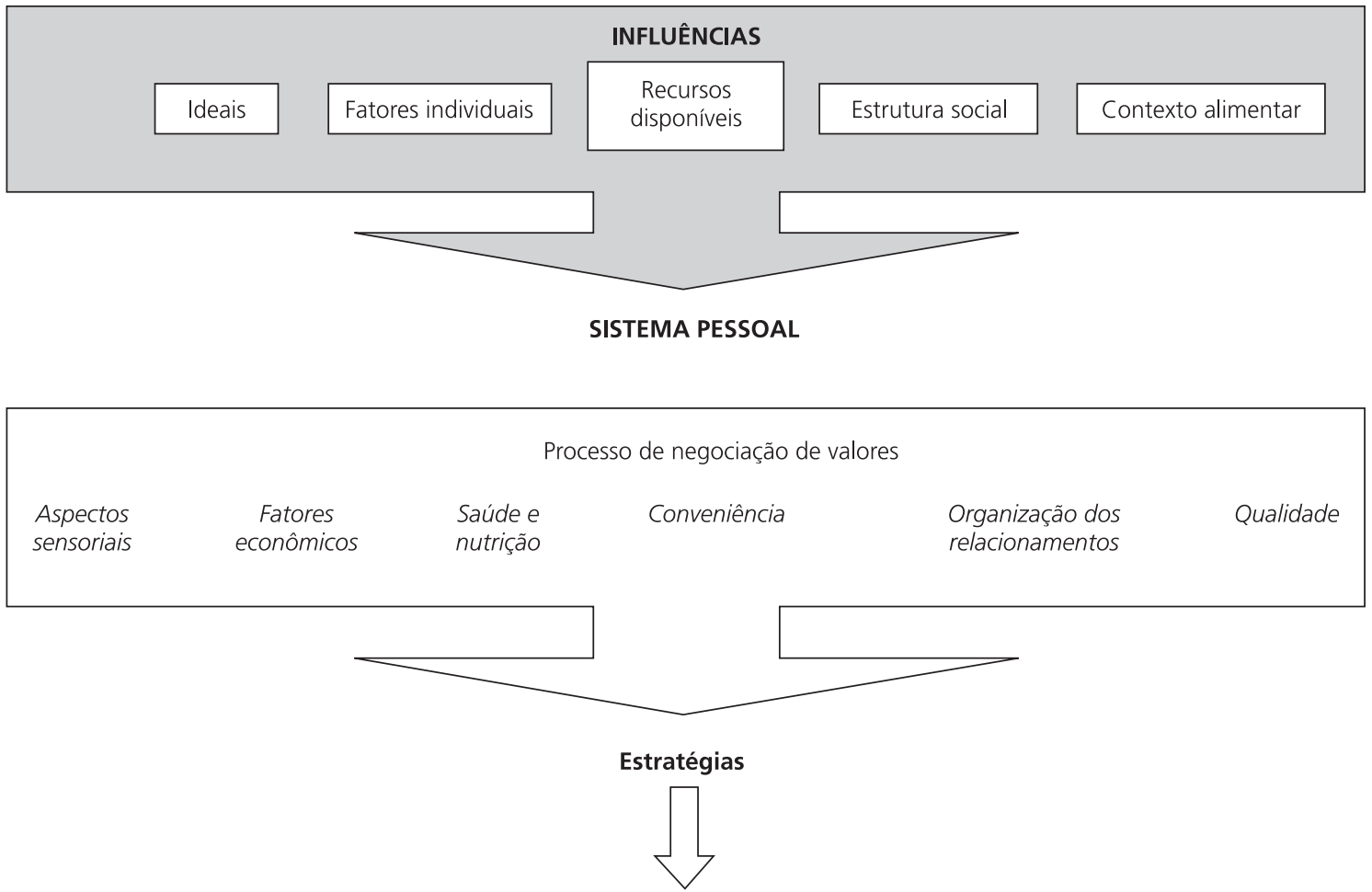

ESCOLHA ALIMENTAR

Figura 1. Modelo do processo de escolha alimentar adaptado de Furst et al. ${ }^{15}$. 
tância dos valores na escolha alimentar dos indivíduos ${ }^{16}$. Esses estudos buscaram fornecer um entendimento teorético do processo da escolha alimentar de um determinado grupo ${ }^{12,16}$.

\section{Variáveis relacionadas aos alimentos}

Em relação aos alimentos, encontraram-se estudos citando alguns determinantes de escolha como a aparência ${ }^{17,18}$, o tipo de preparação ${ }^{19,20}$, sabor $r^{9,17}$, a variedade ${ }^{17,18}$ e 0 valor nutricional ${ }^{1,2,17,19,20}$. Esses aspectos foram referidos pelos autores em diferentes tipos de estudos, aplicados com diversas abordagens, mas possuindo em comum a busca dos motivos que levam o homem a escolher determinado alimento, conforme o demonstrado no Quadro 2.

Um estudo avaliou as influências que levam às escolhas do prato pelos indivíduos que comem fora de casa na cidade do Rio de Janeiro. Para tal avaliação foi desenvolvido um questionário, cujos indicadores utilizados foram: aparência, paladar, variedade, hábito, valor nutricional, preço, saúde e qualidade ${ }^{17}$. A pesquisa foi feita em seis estabelecimentos de diferentes categorias (restaurante por peso, restaurante à la carte, restaurante fast-food, bar e lanchonete e barraca de rua), contando com a participação de 300 indivíduos, abordados na saída de cada estabelecimento (60 indivíduos de cada categoria de estabelecimento). 0 questionário utilizado abordava a atitude dos indivíduos, de acordo com o grau de importância dos atributos citados, em uma escala de "muitíssimo importante" (1) a "nenhuma importância" (5) para a codificação das informações obtidas. Dos fatores citados, a maioria dos entrevistados concordou que a qualidade da refeição, o paladar, a saúde e a variedade são os responsáveis pela escolha do prato nos estabelecimentos avaliados.

Nield et al. ${ }^{18}$ realizaram um estudo para avaliar os indicadores da satisfação dos turistas hospedados em um resort na Romênia em relação aos serviços de alimentação. Foram retornados 341 questionários por 17 grupos. Aplicou-se a análise de variância (ANOVA) para categorizar as diferenças entre os grupos, de acordo com os níveis de satisfação com cada atributo ao serviço de alimentação. E o teste t de Student para investigar se os pontos dos itens de satisfação entre os turistas foram estatisticamente diferentes uns dos outros. Os atributos pontuados em uma escala de valores (de 1 - pobre a 5 - excelente) foram os seguintes, em ordem decrescente de satisfação: qualidade do alimento, preço, variedade dos pratos, atração do ambiente, apresentação dos alimentos, número de pratos (opções), padrão do serviço de alimentação, rapidez do serviço. Esses fatores permitiram avaliar o grau de satisfação desses turistas, como forma de indicar recomendações aos serviços prestados. Entretanto, consideraram-se como limitações do estudo os dados que poderiam influenciar os resultados, listados a seguir: o tamanho da amostra, que divergiu em turistas do Leste e do Oeste Europeu, as diferenças culturais e regionais e as mudanças sazonais. Isso implica na adequação à recomendação de acordo com as diferenças citadas. Para tal, os autores sugerem a reaplicação do estudo em outros locais, para melhor verificar os níveis de satisfação dos turistas com relação aos serviços de alimentação prestados.

Quadro 2. Variáveis relacionadas aos alimentos, de acordo com os autores pesquisados.

\begin{tabular}{|c|c|c|c|c|c|c|}
\hline Variáveis dos alimentos & Castelo Branco ${ }^{9}$ & Nield et al. ${ }^{10}$ & Mills \& Clay ${ }^{11}$ & Poulain ${ }^{12}$ & Gedrich $^{13}$ & Scholderer et al. ${ }^{12}$ \\
\hline Aparência & $x$ & $x$ & & & & \\
\hline Tipos de preparação & & & $x$ & $x$ & & $x$ \\
\hline Variedade & $x$ & $x$ & & & & \\
\hline Sabor & $x$ & & & $x$ & $x$ & \\
\hline Valor nutricional & $x$ & & $x$ & $x$ & & $x$ \\
\hline
\end{tabular}


Mills \& Clay ${ }^{19}$ avaliaram alguns fatores para pesquisar sobre a lei que denominaram de Verdade no Cardápio no momento da escolha dos itens nele contidos, em uma pesquisa envolvendo comensais de restaurantes dos Estados Unidos, os quais são membros de grupos de listas cadastradas. A coleta de dados foi feita por meio eletrônico, pela rede de conexões on-line. 0 questionário, enviado para o endereço eletrônico, continha questões abertas e fechadas. Estas últimas questionavam sobre informações nutricionais dos cardápios, informações da descrição dos itens do cardápio (conhecimento da porção, apresentação visual, quantidade e qualidade dos alimentos, meios de conservação, técnicas de preparo) e informações sobre os ingredientes presentes nos itens do cardápio (a origem e a marca). Esses fatores foram pontuados pelos sujeitos pesquisados dentro de uma escala de quatro pontos, pelos graus de importância, de não-importante a extremamente importante. 0 conhecimento da porção foi considerado o mais importante fator pelos respondentes na escolha dos itens do cardápio. A conclusão do estudo foi que as informações contidas nos cardápios devem ser completas e acuradas, abrangendo informações nutricionais, tamanho da porção, método de preparo, entre outras questões de seguridade de produtos alimentícios, uma vez que elas são consideradas um meio pelo qual os freqüentadores desses restaurantes se utilizam para fazerem suas escolhas. Os autores salientam que o estudo é limitado pelo fato de a população pertencer aos membros das listas pesquisadas, devendo ser expandida a participantes fora da rede eletrônica de comunicação, diretamente nos restaurantes.

Scholderer et al. ${ }^{20}$ testaram e validaram um instrumento de estilo de vida alimentar relatado (FRL - food-related lifestyle) em diferentes culturas do Oeste Europeu (Dinamarca, França, Alemanha, Espanha e Reino Unido), que se baseava em três dimensões: atitudes, interesses e opiniões. 0 questionário consistiu em 69 itens medindo 23 dimensões dentro dos cinco domínios citados. Cada dimensão mediu três itens em uma escala de sete pontos, que compreendiam valores desde "discordo completamente" (1 ponto) até "concordo completamente" (7 pontos). Com a análise do FRL concluiu-se que as medidas do instrumento têm as mesmas construções, inter-relações e, variabilidade entre os países. As propriedades de medida dos domínios - técnicas de preparo, aspectos qualitativos, situações de consumo e motivos de compra (considerado importante para medir diferenças individuais específicas em relação aos valores pessoais sobre 0 alimento) - foram invariáveis( ${ }^{(6)}$ durante as aplicações do instrumento. Esses resultados demonstram que o FRL provê medidas consistentes das culturas ocidentais européias, cujos itens relatados podem ser citados como variáveis relacionadas aos alimentos.

\section{Os métodos de avaliação da escolha alimentar}

Na literatura científica encontram-se alguns conceitos relativos ao estilo de vida no contexto alimentar. Nesse aspecto, Grunert ${ }^{21}$ discorre sobre essa abordagem, se referindo ao conceito cognitivo do comportamento do consumidor, explicando que 0 estilo de vida é construído mentalmente, mas não é idêntico ao comportamento atual. Dessa forma, a escolha alimentar do consumidor é direcionada pela sua expectativa, ou seja, pelo valor dado ao alimento ou, por outro lado, pelo grau de liberdade dado ao indivíduo para realizar essas escolhas, conferindo a possibilidade de diferentes estilos de vida, relatados no contexto alimentar. Essas diferenças puderam ser relacionadas às categorias cognitivas mencionadas (situações de compra, atributos do produto mais desejado, forma de preparação da refeição, hábito alimentar, conseqüências desejadas) relacionadas tanto

\footnotetext{
${ }^{6} 0$ termo "invariável" é utilizado na área de Estatística para designar "al go que não variou", conforme os testes estatísticos aplicados (Exemplos: Análise Fatorial Confirmatória - Confirmatory Factor Analysis, mede níveis de medida de "invariâncias"), demonstrados por Scholderer et al. ${ }^{12}$
} 
aos produtos alimentícios em si quanto aos valores individuais.

Alguns estudos permitem analisar os métodos utilizados para avaliar a escolha alimentar de um determinado grupo. Nesse contexto, Stepoe et al. ${ }^{22}$ escreveram sobre o desenvolvimento de um instrumento de medida dos motivos relatados à escolha alimentar, denominado Questionário de Escolha Alimentar (FCQ - Food Choice Questionary). Esse questionário baseou-se em extensa literatura, sugerindo a avaliação da percepção individual desses fatores, em um contexto multidimensional da escolha alimentar. Esse instrumento englobou nove fatores que levam à escolha alimentar: saúde (controle do peso e doenças); conveniência da aquisição e preparação dos alimentos; aspectos sensoriais (odor, sabor e aparência); preço (fator econômico); conteúdo natural (preocupação com o uso de aditivos e alimentos naturais); humor (fator emocional); familiaridade (hábitos alimentares); preocupação ética (fatores ambientais e políticos socialmente aceitáveis), e controle de peso (destacado separadamente do fator saúde como fator de preferência estético-cultural). Os quatro primeiro fatores são considerados como mais importantes nos estudos de validação e reprodutibilidade do questionário. Algumas limitações, como o fator cultural, são destacadas, tornando o instrumento válido especificamente à população do estudo (população urbana ocidental).

O FCQ possibilita a oportunidade de avaliar os fatores relevantes da escolha alimentar percebidos individualmente pela população urbana ocidental. Esses fatores, entretanto, não se referiram, necessariamente, ao comportamento real de escolha alimentar, ou seja, às práticas alimentares realizadas por esses indivíduos, mas sim às representações dos motivos que levam a essas escolhas.

Outra forma de avaliar as práticas alimentares dos indivíduos é a utilização de inquéritos dietéticos. Entretanto, a técnica de observação dessas práticas permite verificar as possíveis discrepâncias existentes entres as práticas declaradas e as práticas observadas ${ }^{5}$. Garcia ${ }^{23}$ aborda as limitações desses inquéritos e a necessidade de os combinar para reduzir tal viés. Nessa ótica, verifica-se a existência de instrumentos que avaliam o consumo alimentar e que servem de base para avaliar as escolhas alimentares, mas que, sozinhos, não suportam a avaliação dessas escoIhas. Isso se dá pela complexidade da escolha alimentar, a qual apresenta uma série de determinantes, que estão relacionados à percepção e à representação do comedor humano em relação ao alimento. Nesse sentido, torna-se viável utilizar procedimentos metodológicos complementares de avaliação da escolha alimentar, baseando-se em estudo teórico sobre seus determinantes.

Nessa direção, a abordagem metodológica utilizada por Poulain ${ }^{1}$ é baseada na avaliação das práticas alimentares declaradas, comparadas às práticas alimentares observadas. As práticas alimentares declaradas são definidas como a idéia que os indivíduos possuem em relação à refeição apropriada, às opiniões, às atitudes e aos valores, transmitid os oralmente. Já as práticas observadas consistem em verificar as práticas alimentares atuais, que os indivíduos realizam e que são passíveis de observação ${ }^{1,4,5}$. Esses conceitos permitiram a utilização de um método, em que as práticas declaradas foram investigadas por meio de entrevistas conduzidas após o almoço, a partir de um protocolo e da aplicação de um questionário sobre o serviço prestado. Para concretizar a avaliação das práticas observadas, realizou-se a observação direta da bandeja, na entrada ou já no refeitório, evitando interferências no momento da escolha alimentar. Nessa abordagem é possível identificar como o comensal se comporta perante os alimentos, mas, muitas vezes, esses comportamentos escapam às questões relatadas por ele próprio.

Dentro dessa perspectiva, a escolha alimentar pode ser encarada por aspectos múltiplos passíveis de avaliação, ao realizar o cruzamento dos dados obtidos pela aplicação desse método. Contudo, o mesmo autor analisou as discrepâncias existentes entre as práticas alimentares declaradas e as práticas alimentares observadas para verificar a lógica da escolha alimentar. Para suas análises 
aplicaram-se diferentes testes estatísticos que permitiram quantificar as respostas, de forma a verificar a significância das associações realizadas ${ }^{1}$. Deve-se levar em consideração que esses estudos são epidemiológicos, de base populacional, e os seus dados podem ser generalizados. No Brasil essa abordagem metodológica foi adotada em um estudo sobre a escolha alimentar com enfoque nos comensais de restaurante por peso ${ }^{24}$.

Pode-se referir outras abordagens relacionadas à avaliação dos fatores que levam à escolha alimentar. A equipe da Divisão de Ciências Nutricionais (Division of Nutritional Sciences) da Universidade de Cornell (Cornell University) de Nova lorque, nos EUA, desenvolve uma linha de pesquisa relacionada com a escolha alimentar, demonstrada em alguns estudos $3,12,15,16$.

Esses estudos empregaram um método qualitativo e um paradigma construtivista para coleta e análise dos dados, para explicar o processo de escolha alimentar dos diferentes grupos estudados. A coleta foi feita por entrevistas aprofundadas semi-estruturadas, gravadas e transcritas. A análise envolveu a codificação das entrevistas transcritas para conceitos gerais que surgiram dessas entrevistas no estudo de Falk et al. ${ }^{3}$, enquanto nos outros $\mathrm{s}^{3,15,16}$ utilizou-se o método comparativo de Strauss \& Corbin ${ }^{25}$ das categorias e dos temas encontrados e codificados pela transcrição das entrevistas.

Verificou-se a associação entre alguns fatores de escolha alimentar (os ideais, as identidades e as representações dos papéis de cada um nas diferentes culturas) dos indivíduos avaliados, no estudo de Devine et al. ${ }^{15}$, e a mudança de contextos ou situações, os quais são caracterizados pelo espaço, tempo e relacionamentos.

No estudo de Connors et al. ${ }^{16}$, os principais valores relativos ao alimento relatado descritos pelos indivíduos foram cinco: a saúde, o sabor, 0 custo, a relação tempo/conveniência e a organização dos relacionamentos. A repetição desses valores de negociação, expressando um sistema pessoal alimentar, permitiu chegar às estratégias de escolha alimentar dependente de diferentes situações dos indivíduos estudados. Esse sistema pessoal alimentar contribuiu para estruturar um conceito teórico sobre a escolha alimentar. Já Falk et al. ${ }^{12}$ enfocaram a avaliação nas interpretações dos indivíduos sobre suas experiências de escolha alimentar relacionadas à saúde. Os achados identificaram como as pessoas organizam e constroem significados para o conceito de saúde relacionado aos alimentos, e como elas aplicam isso aos seus comportamentos alimentares. Encontraram-se sete grandes temas relacionando saúde e alimentação, que consistiram em uma série de crenças complexas, agregando temas secundários. Entretanto, esse sistema de crenças de cada participante não pode ser considerado completo para verificar diretamente suas crenças sobre saúde, uma vez que as suas descrições emergiram de questões relacionadas à escolha e ao comportamento alimentar.

A conclusão dos estudos citados foi que a perspectiva em relação ao método empregado se direciona ao melhor entendimento do processo de escolha alimentar, implicando no desenvolvimento de uma teoria desse processo. Essa conclusão se deu pelo fato de o método proporcionar 0 surgimento das categorias de análise, importantes na definição de alguns dos fatores que determinam a escolha alimentar dos indivíduos, dentro do processo referido no modelo ilustrado por esses estudos.

\section{CONSIDERACÕ ES FINAIS}

Diante do exposto, parece ser possível refletir so bre os meios para avaliar a escolha alimentar humana e os direcionar a uma análise efetiva e coerente, buscando elucidar a complexidade dessas questões. Assim, a junção das abordagens quantitativas e qualitativas de estudo pode contribuir para dar maior suporte à pesquisa sobre escolha alimentar. No primeiro caso, como foi referido, serve para iniciar uma abordagem metodológica que possibilita reduzir alguns vieses, já citados anteriormente. Com relação à abordagem qualitativa de estudo, fica evidente 0 aprofundamento na análise dos dados, pela qual se podem obter categorias da escolha alimentar que servirão 
para embasar o cruzamento quantitativo dos dados e contribuir para uma discussão mais consistente desses dados.

Diante disso, os métodos empregados em estudos da área das Ciências Humanas podem contribuir para as discussões nas Ciências Nutricionais, inclusive na questão da escolha alimentar. Isso permite o cruzamento de olhares e a interdisciplinaridade, aprofundando os conhecimentos sobre o comedor humano.

\section{COLABORADORES}

M.M. JOMORI, R.P.C. PROENÇA E M.C.M. CALVO participaram da concepção e redação do artigo.

\section{REFERÊ N CIAS}

1. Poulain J-P. The contemporary diet in France: "de-structuration" or from commensalisms to "vagabond feeding". Appetite. 2002; 39(1):43-55.

2. Poulain J-P. Sociologias da alimentação. Florianópolis: Editora UFSC; 2004.

3. Falk LW, Sobal J, Bisogni CA, Connors M, Devine $C M$. Managing health eating: definitions, classifications and strategies. Health Educ Behav. 2001; 28(4):425-39.

4. Poulain JP, Proença RPC. O espaço social alimentar: um instrumento para o estudo dos modelos alimentares. Rev Nutr. 2003; 16(3):245-56.

5. Poulain JP, Proença RPC. Reflexões metodológicas para o estudo das práticas alimentares. Rev Nutr. 2003; 16(4):365-86.

6. Fischler C. L'Homnivore. Le goût, la cuisine et le corps. Paris: Éditions Odile Jacob; 1990.

7. Courbeau J-P, Poulain J-P. Libres mangeurs? In: Courbeau J-P, Poulain J-P, editors. Penser I'alimentation: entre imaginaire et rationalité. Toulouse: Éditions Privat; 2002. p.137-56.

8. Bourdieu P. La distinction: critique sociale du jugement. Paris: Edítions de M inuit; 1979.

9. Gedrich K. Determinants of nutritional behavior: a multitude of levers for successful intervention? Appetite. 2003; 41(3):231-8.

10. Fantino M. Plasir et prise alimentaire: aspects physiologiques. Cah Nutr Diét. 1999; 34(3):149-52.

11. Furst T, Connors M., Bissogni CA, Sobal J, Falk LW. Food choice: a conceptual model of the process. Appetite. 1996, 26(3):247-66.
12. Falk LW, Bisogni CA, Sobal J. Food choice process in older adults: a qualitative investigation. J Nutr Educ. 1996; 28(5):257-65.

13. Tse $A C B, \operatorname{Sin} L$, Yin FHK. How a crowded restaurant affects consumers' attribuition behavior. Hospit Manag. 2002; 21(4):449-54.

14. Batalha MO, Luchese T, Lambert JL. Hábitos de consumo alimentar no Brasil: realidade e perspectivas. In: Batalha M O. Gestão de agronegócios: textos selecionados. São Carlos: Editora UFSCar; 2005.

15. Devine CM, Sobal J, Bisogni CA, Connors M. Food choices in three ethnic groups: Interactions of ideals, identities, and roles. J Nutr Educ. 1999; 33(2): 6-93.

16. Connors M, Bisogni CA, Sobal J, Devine CM. Managing values in personal food systems. Appetite. 2001; 36(3):189-200.

17. Castelo Branco NSD. Análise da alimentação fora do domicílio de consumidores do centro comercial do Município do Rio de Janeiro - RJ [tese]. Campinas: Universidade Estadual de Campinas; 2000.

18. Nield K, Kozak M , Legrys G. The role of food service in tourist satisfaction. Hospit Manag. 2000; 19(4): 375-84.

19. Mills JE, Clay JM. The truth-in-menu law and restaurant consumers. Foodserv Res Int. 2001; 13:69-82.

20. Scholderer J, Brunso K, Bredahl L, Grunert KG. Cross-cultural validity of the food-related lifestyles instrument (FRL) within Western Europe. Appetite. 2004; 42(2):197-211.

21. Grunert KG. Towards a concept of food-related life style. Appetite. 1993; 21(2):151-5.

22. Stepoe A, Pollard TM., Wardle J. Development of a mesure of the motives underlying the selection of food: the food choice questionnaire. Appetite. 1995; 25(3):267-84.

23. Garcia RWD. A comida, a dieta, o gosto: mudanças na cultura alimentar urbana [tese]. São Paulo: Universidade de São Paulo; 1999.

24. Jomori MM. Escolha alimentar do comensal de um restaurante por peso [dissertação]. Florianópolis: Universidade Federal de Santa Catarina; 2006.

25. Strauss AL, Corbin J. Basics of qualitative research: grounded theory procedures and research. New-bury Park (CA): Sage; 1990.

Recebido em: 12/4/2007

Versão final reapresentada em: 17/9/2007 Aprovado em: 2/1/2008 
